This article was downloaded by: [University of New Mexico]

On: 19 January 2011

Access details: Access Details: [subscription number 792947466]

Publisher Taylor \& Francis

Informa Ltd Registered in England and Wales Registered Number: 1072954 Registered office: Mortimer House, 3741 Mortimer Street, London W1T 3JH, UK

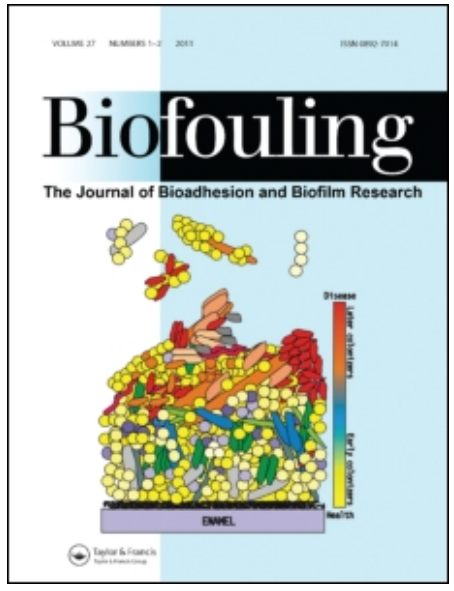

\title{
Biofouling
}

Publication details, including instructions for authors and subscription information:

http://www.informaworld.com/smpp/title content=t713454511

\section{Characterization and effect of biofouling on polyamide reverse osmosis and nanofiltration membrane surfaces}

Mohiuddin Md. Taimur Khan ${ }^{\mathrm{a}}$; Philip S. Stewartt; David J. Mollc; William E. Mickols ${ }^{\mathrm{d}}$; Sara E. Nelson;

Anne K. Camper

${ }^{a}$ Center for Molecular Discovery, MSC08 4640, University of New Mexico, Albuquerque, NM, USA ${ }^{b}$

Center for Biofilm Engineering and Department of Chemical and Biological Engineering, Montana

State University, Bozeman, MT, USA c The Dow Chemical Company, Larkin Laboratory, Midland, MI,

USA ${ }^{d}$ The Dow Chemical Company, FilmTec R \& D, Edina, MN, USA ${ }^{\mathrm{e}}$ Institute for Systems Biology,

Seattle, WA, USA ${ }^{\mathrm{f}}$ Center for Biofilm Engineering and Department of Civil Engineering, Montana

State University, Bozeman, MT, USA

First published on: 19 January 2011

To cite this Article Khan, Mohiuddin Md. Taimur, Stewart, Philip S. , Moll, David J. , Mickols, William E. , Nelson, Sara E. and Camper, Anne K.(2011) 'Characterization and effect of biofouling on polyamide reverse osmosis and nanofiltration membrane surfaces', Biofouling, 27: 2, 173 - 183, First published on: 19 January 2011 (iFirst)

To link to this Article: DOI: $10.1080 / 08927014.2010 .551766$

URL: http://dx.doi.org/10.1080/08927014.2010.551766

\section{PLEASE SCROLL DOWN FOR ARTICLE}

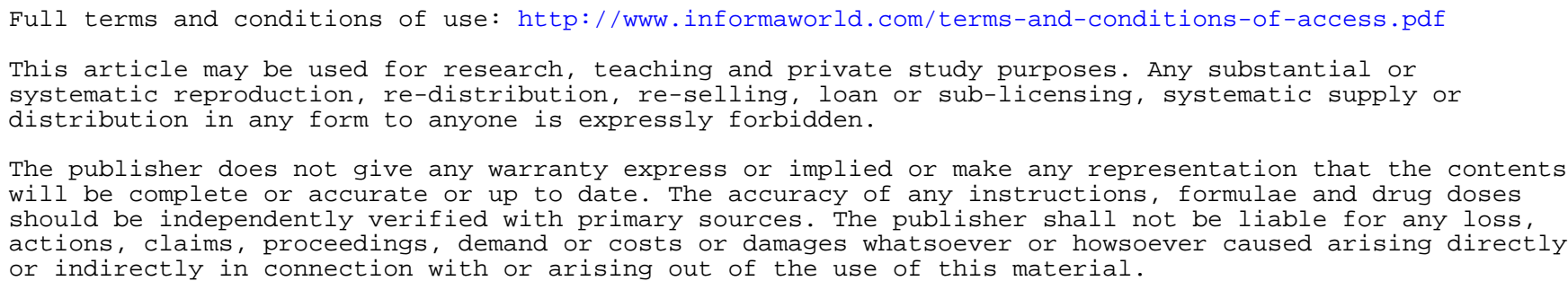




\title{
Characterization and effect of biofouling on polyamide reverse osmosis and nanofiltration membrane surfaces
}

\author{
Mohiuddin Md. Taimur Khan ${ }^{\mathrm{a} *}$, Philip S. Stewart ${ }^{\mathrm{b}}$, David J. Moll ${ }^{\mathrm{c}}$, William E. Mickols ${ }^{\mathrm{d}}$, Sara E. Nelson ${ }^{\mathrm{e}}$ and \\ Anne K. Camper ${ }^{\text {f* }}$ \\ ${ }^{a}$ Center for Molecular Discovery, MSC08 4640, University of New Mexico, Albuquerque, 87131-0001 NM, USA; ${ }^{b}$ Center for \\ Biofilm Engineering and Department of Chemical and Biological Engineering, Montana State University, Bozeman, MT 59717- \\ 3980, USA; ${ }^{c}$ The Dow Chemical Company, Larkin Laboratory, 1691 North Swede Road, Midland, 48674 MI, USA; ${ }^{d}$ The Dow \\ Chemical Company, FilmTec $R \&$ \& , 5230 73rd St, Edina, MN 55439, USA; ${ }^{e}$ Institute for Systems Biology, 1441 N. 34 th St, \\ Seattle, WA 98103-8904, USA; ${ }^{f}$ Center for Biofilm Engineering and Department of Civil Engineering, Montana State University, \\ Bozeman, MT 59717-3980, USA
}

(Received 20 June 2010; final version received 25 December 2010)

\begin{abstract}
Biofouling is a major reason for flux decline in the performance of membrane-based water and wastewater treatment plants. Initial biochemical characterization of biofilm formation potential and biofouling on two commercially available membrane surfaces from FilmTec Corporation were investigated without filtration in laboratory rotating disc reactor systems. These surfaces were polyamide aromatic thin-film reverse osmosis (RO) (BW30) and semiaromatic nanofiltration (NF270) membranes. Membrane swatches were fixed on removable coupons and exposed to water with indigenous microorganisms supplemented with $1.5 \mathrm{mg}^{-1}$ organic carbon under continuous flow. After biofilms formed, the membrane swatches were removed for analyses. Staining and epifluorescence microscopy revealed more cells on the RO than on the NF surface. Based on image analyses of 5- $\mu$ m thick cryo-sections, the accumulation of hydrated biofoulants on the RO and NF surfaces exceeded 0.74 and $0.64 \mu$ m day $^{-1}$, respectively. As determined by contact angle the biofoulants increased the hydrophobicity up to $30^{\circ}$ for RO and $4^{\circ}$ for $\mathrm{NF}$ surfaces. The initial difference between virgin RO and NO hydrophobicities was $\sim 5^{\circ}$, which increased up to $25^{\circ}$ after biofoulant formation. The initial roughness of RO and NF virgin surfaces ( $75.3 \mathrm{~nm}$ and $8.2 \mathrm{~nm}$, respectively) increased to $48 \mathrm{~nm}$ and $39 \mathrm{~nm}$ after fouling. A wide range of changes of the chemical element mass percentages on membrane surfaces was observed with X-ray photoelectron spectroscopy. The initial chemical signature on the NF surface was better restored after cleaning than the RO membrane. All the data suggest that the semi-aromatic NF surface was more biofilm resistant than the aromatic RO surface. The morphology of the biofilm and the location of active and dead cell zones could be related to the membrane surface properties and general biofouling accumulation was associated with changes in the surface chemistry of the membranes, suggesting the validity of the combination of these novel approaches for initial assessment of membrane performance.
\end{abstract}

Keywords: reverse osmosis and nanofiltration membranes; biofouling; surface hydrophobicity and roughness; biofilm morphology; live and dead cells

\section{Introduction}

Reverse osmosis (RO) and nanofiltration (NF) membranes have been increasingly used to produce freshwater in wastewater reuse, brackish/seawater purification, and in the drinking water treatment industries. However, the impairment of RO and NF membrane performance originating from multi-fouling processes creates unavoidable threats to these industries (Burashid and Hussain 2004; Chen et al. 2004; Belfer et al. 2005; Xu et al. 2006; Kaufman et al. 2010). The major types of fouling in RO and NF membranes are inorganic salt precipitation, organic, colloidal and particulate matter, and microbiological (usually governed by bacterial biofilm formation) (Flemming 1997;
Schneider et al. 2005; Herzberg and Elimelech 2007). The accumulation of dissolved solutes and macromolecules near or on the surface of the membrane causes concentration polarization effects (Vrijenhoek et al. 2001) and a subsequent accumulation of retained materials in the boundary layer above the membrane leads to flux decline (Manttari et al. 2000; Zularisama et al. 2006).

Bacteria in natural and engineered aquatic systems are ubiquitous, and the subsequent formation of biofilm biomass consisting of structured microbial communities and their byproducts is inevitable (Costerton et al. 1995; Flemming et al. 1997; Donlan 2002; Stoodley and Stoodley 2002). Colloidal and particulate

*Corresponding authors. Email: mmtkhan@salud.unm.edu; anne_c@biofilm.montana.edu Published online 19 January 2011 
fouling can potentially be reduced using pretreatment options (Ridgway and Flemming 1996); however, in practice, removing all microorganisms is not possible and the few that pass the barrier of the pretreatment system can adhere to the membrane, grow and form biofilm, which affects system performance. Fouling requires frequent chemical cleaning, which ultimately shortens membrane life, thus increasing the operational and maintenance costs of RO and NF membrane plants. Furthermore, biofouling can alter the surface chemistries, narrowing down the flow channels across the RO and NF surfaces (Kwak and Ihm 1999; Khan et al. personal observations). It is reasonable to assume that biofouling of RO and NF membranes is ubiquitous and it is important to deal with this process rather than expect that it can be prevented.

Biofouling is the least understood and controlled fouling mechanism. Even though much has been done to understand the principles of biofilm formation, the dynamics of RO and NF membrane biofouling are relatively unexplored. At a fundamental level, several studies to investigate the organisms and mechanisms of membrane biofouling have been carried out and these include terminal restriction fragment length polymorphism (T-RFLP) fingerprinting (Pang and Liu 2007), PCR-amplified 16S rRNA clone libraries (Ivnitsky et al. 2005), and culture based methods (Ridgway and Flemming 1996) for community analysis; laser scanning confocal microscopy and environmental scanning electron microscopy observation for Pseudomonas aeruginosa PA01 biofouling (Herzberg and Elimelech 2007); motility and chemotaxis assays (Herzberg and Elimelech 2008); biofilm EPS extraction, protein and polysaccharide measurements (Herzberg et al. 2009); adenosinetriphosphate (ATP), total direct cell count (TDC), and heterotrophic plate counts (HPC) (Vrouwenvelder and van der Kooij 2001); and direct light microscope observations during $\mathrm{RO}$ and $\mathrm{NF}$ biofilm formation and estimation of cell and membrane interfacial free energy (Subramani and Hoek 2008). These studies have provided useful qualitative information on RO membrane flux decline, but most were performed using either specific bacteria or for the identification of specific groups of organisms in the biofouled membrane and did not provide detailed information on biofoulant structure.

In addition, several fundamental investigations on membrane fouling have explored the effects of membrane surface properties such as pore size distribution, surface roughness and structure, electrokinetic (zeta potential) characteristics, chemical properties (hydrophobic/ hydrophilic), and specific chemical structure on fouling and subsequent flux decline and polarization effects (Zhu and Elimelech 1997; Riedl et al. 1998). However, none of these studies quantified the biofoulants or correlated their structures and distributions on RO and NF surfaces with simultaneous changes in the chemical properties and hydrophobicity of the surface. A suite of biomass parameters and analytical tools are available, but the question remains which biofouling parameter(s) and tool(s) are needed to assess biofouling on RO and NF surfaces and its impacts on these surfaces and their performances.

The objectives of this research were: (1) to develop methods for obtaining reproducible membrane biofilms in terms of cellular distribution and biomass thickness and provide quantitative measurements and visualization of biofoulants on RO and NF membranes, and (2) correlate biofoulant structure with membrane property changes and quantify changes in the chemical properties of the membrane surface due to biofouling formation. The short-term, accelerated biofouling experiments used without flux allowed the selection and evaluation of methods to elucidate the fundamental effects of biofouling on $\mathrm{RO}$ and $\mathrm{NF}$ membrane surfaces in a laboratory setting.

\section{Materials and methods}

\section{Membrane type}

To observe biofilm formation and its impact on RO and NF membrane surfaces, commercially available polyamide aromatic thin-film composite RO (Dow BW30) and semi-aromatic, weakly acidic $\mathrm{COO}^{-}$ groups and piperazine based polyamide NF (Dow NF270) membranes (FilmTec Corporation, Dow Chemical Company, USA) were examined. Detailed information about the heterogeneity and chemistry of these surfaces and of the aromatic and semi-aromatic groups on RO and NF surfaces, respectively, is proprietary. The general performances of these surfaces are that at $125 \mathrm{psi}$ applied pressure and $2000 \mathrm{ppm}$ initial concentration of $\mathrm{NaCl}$ in DI water, the membrane productivity and water permeability coefficient $/ \%$ salt passage (A-value $/ \% \mathrm{SP}$ ) ratios were $32 \mathrm{gfd}$ $\left(5.4 \mathrm{~cm} \mathrm{~h}^{-1}\right)$ and $45 \mathrm{gfd}\left(7.6 \mathrm{~cm} \mathrm{~h}^{-1}\right)$, respectively and 0.16 and 0.37 , respectively, for RO and NF membranes. The $\mathrm{NaCl}$ rejection was $\sim 99.6 \%$ for $\mathrm{RO}$ and $\sim 99 \%$ for NF surfaces.

\section{Reactor setup}

RO and NF membrane swatches were cut from flat sheet rolls and boiled in nanopure water for $10 \mathrm{~min}$ (according to the manufacturer's instructions) to remove residual chemicals and any surface contamination. Initial results showed that this process did not change the surface structures and chemistries of these membranes. After cooling to room temperature and 
prior to use, the membrane swatches were gently washed several times with nanopure water. The membrane swatches were adhered to removable polycarbonate coupons $(12.7 \mathrm{~mm}$ in diameter and six per reactor) with silicon rubber sealant (Dow Corning, USA). Once the membrane swatches were fixed on the coupons, they were placed in the rotor of the rotating disc reactors (RDR) (Biosurface Technologies Corporation, USA). Briefly, these systems have a variable speed rotating disk, an operating volume of $250 \mathrm{ml}$, and a high surface area to volume ratio. The advantage of these systems is that the fluid conditions can be set and maintained so that artifacts introduced by varying shear stress or residence time can be eliminated. Moreover, in these systems well-defined hydrodynamic conditions and retention time on biofilm can be maintained. The rotation speed of the rotor in the RDR was $50 \mathrm{rpm}$ and the hydraulic retention time was $3.5 \mathrm{~h}$. The temperature was ambient $\left(25^{\circ} \mathrm{C}\right)$ and $\mathrm{pH}$ was $7.5 \pm 0.5$.

The reactors were fed continuously with Bozeman (Montana, USA) tap water that flowed through a granular activated carbon (GAC) column and a biologically activated carbon (BAC) column operated in up-flow mode as a source of indigenous microorganisms. These columns removed the majority of the background carbon from the tap water. The details of Bozeman tap were described elsewhere (Khan et al. 2010b). To enhance the growth of biofilm, the biologically treated water was amended with nutrients $(\mathrm{C}: \mathrm{N}: \mathrm{P})$ (equimolar concentrations of glutamic acid, glucose, galactose and arabinose were used for a carbon source and $\mathrm{KNO}_{3}$ and $\mathrm{K}_{2} \mathrm{HPO}_{4}$ were used for nitrogen and phosphorous sources, respectively). These constituents $(\mathrm{C}, \mathrm{N}$, and $\mathrm{P}$ ) were added to the reactors at a 100:10:1 (molar ratio) basis. Specifically, $5.54 \mathrm{ml}$ of carbon source, $16.88 \mathrm{ml}$ of nitrogen source, and $4 \mathrm{ml}$ of phosphorus source were added to a 20-1 container of autoclaved nanopure water.
The operating conditions and analyses are shown in Table 1. Each reactor was operated continuously for 31 days in Phase-I and 28 days in Phase-II to ensure good biofilm growth. Imaging of live and dead cells as determined by differential staining of membranes and cryo-sectioning assays of membrane biofilm were repeated in Phase-II (second experiment) with intermediate samplings to verify the reproducibility of biofoulant formation on the surfaces as calculated from the replicate experiments. Colony forming units $(\mathrm{CFU})$ in the BAC water were measured in triplicate using R2A agar (Fisher Scientific, IL) spread plates incubated at room temperature for 7 days. After colonization, membranes were carefully taken off from the coupons using a sterilized razor blade and hemostat without disturbing the biofilm. The RO and NF swatches with biofoulants were cut into pieces with sterilized scissors for the assays described in Table 1 .

\section{Visualization and quantification of membrane biofoulants}

The Live/Dead BacLight ${ }^{\mathrm{TM}}$ bacterial viability kit for microscopy and quantitative assay (Invitrogen/Molecular Probes, Oregon) was used for staining live and dead cells on the surfaces of the RO and NF membranes. Equal amounts $\left(1.5 \mu \mathrm{l} \mathrm{ml}^{-1}\right)$ of SYTO $9(3.34 \mathrm{mM})$ and propidium iodide $(20 \mathrm{mM})$ dyes were diluted in $1 \mathrm{ml}$ of nanopure water and after proper dilution and vortexing, dyes were added to the top surface of the membrane and incubated for $1 \mathrm{~h}$ in the dark. After incubation, the excess dye was carefully washed off with nanopure water and the stained cells were observed under an epifluorescence microscope (Nikon, Eclipse E 800, Japan) with a $100 \times$ objective. Here, according to generally accepted convention, the cells with damaged cell membranes are called dead cells (Khan et al. 2010a).

Membranes are called dead cells (Khan et al. 2010a). Fifteen to 20 images of live and dead cells in

Table 1. Operating conditions of the rotating disc reactors and biofouling assays used in replicate experiments (Phase-I and Phase-II).

\begin{tabular}{|c|c|c|}
\hline Operating conditions & Phase-I & Phase-II \\
\hline Period of run & 31 days & 28 days \\
\hline Sampling frequency & At the end of run & Once a week \\
\hline $\begin{array}{l}\text { Average CFU ml } \\
\text { activated carbon (BAC) treated water }\end{array}$ & $(1.35 \sim 1.9) \times 10^{4}$ & $(1.41 \sim 2.01) \times 10^{4}$ \\
\hline Membrane assays & $\begin{array}{l}\text { Images of live/dead cells, } \\
\text { cryo-sectioning, and AFM } \\
\text { on Day-31 }\end{array}$ & $\begin{array}{l}\text { Images of live/dead cells and } \\
\text { cryo-sectioning on Day- } 8 \text { and } \\
\text { Day-21 (reproducibility of Phase-I); } \\
\text { and X-ray photoelectron spectroscopy } \\
\text { (XPS) and Contact angle on } \\
\text { Dav-14 and Dav-28 }\end{array}$ \\
\hline Nutrient condition $(\mathrm{C}: \mathrm{N}: \mathrm{P})$ & \multirow{3}{*}{\multicolumn{2}{|c|}{$\begin{array}{c}1.5 \mathrm{mg} \mathrm{l}^{-1} \text { of } \mathrm{C}: 0.18 \mathrm{mg} \mathrm{l}^{-1} \text { of } \mathrm{N}: 0.04 \mathrm{mg} \mathrm{l}^{-1} \text { of } \mathrm{P} \\
42 \% \text { of total influent flow-rate } \\
58 \% \text { of total influent flow-rate }\end{array}$}} \\
\hline Flow rate of nutrient & & \\
\hline Flow rate of BAC treated water & & \\
\hline
\end{tabular}


the top layer of biofilm on the membrane surfaces were captured and analyzed with MetaMorph software (Molecular Devices Corporation, USA).

For cryo-sectioning, the RO and NF membrane swatches with biofoulants were stained without disturbing the biofilm using the same procedures above. Detailed procedures are described elsewhere (Khan et al. 2010b). Briefly, a cryostat (Leica CM 1850, Germany) was used to cut sections of $5.0 \mu \mathrm{m}$ thickness. The sectioned layer of the biofoulants and membranes was placed on a positively charged microscope slide (Fisher Scientific, IL) and observed and imaged under the epifluorescence microscope (Nikon, Eclipse E 800, Japan) with a $20 \times$ objective.

The cryo-sections of stained biofilms on membranes were analyzed with MetaMorph software to calculate the regions of live and dead cells and the thickness of accumulated biofoulants on the membrane surfaces. Each image has a distribution of live (green) and dead (red) cells along the thickness of biofilm on the membrane. A line scan was done with the MetaMorph software across the slices of membranes with biofoulants. Each line scan generated two profiles depending on the location of live and dead cells inside the biofilms. To calculate the thickness of live and dead cell regions, the width at the mid height of each distribution was measured. Using the width at mid height of each profile, the software calculated the thickness of each profile in terms of live and dead cell regions. For each membrane swatch, 8-10 slices of membranes were imaged (three images per slice) and from each image, five different line-scans were generated for analysis.

\section{Diagnosis of membrane surface properties}

For atomic force microscopy (AFM) analysis, the RO and NF swatches with and without biofilm (after physical cleaning) were used after drying at room temperature. Parallel control membranes that had not been subjected to biofouling were also analyzed. In the presence of biofoulants on $\mathrm{RO}$ and $\mathrm{NF}$ membranes, the surface morphology and roughness, which are direct measures of surface heterogeneity, were determined using AFM imaging and analysis (Multi-Mode AFM, Digital Instruments, Santa Barbara, CA). Additional samples were analyzed after cleaning to assess how residual biofoulants altered the surface heterogeneity. Imaging was performed in tapping mode with an etched silicon probe (TESP, Digital Instruments, Santa Barbara, CA). The root mean square (RMS) averages of height deviations of roughness (Rq) were measured from the mean image data plane using Equation (1) and expressed as:

$$
\mathrm{Rq}=\sqrt{\frac{\sum Z_{i}^{2}}{N}}
$$

where $Z$ is the maximum vertical distance between the highest and lowest data points in the image prior to the plane-fit, $i$ the particular number of data points and $N$ the total number of data points. The average RMS values of $\mathrm{Rq}$ were calculated from 10 line-scans on each AFM image. For each sample, five AFM images were taken and in each case the effective area was $25 \mu \mathrm{m}^{2}$.

For contact angle measurement and X-ray photoelectron spectroscopy (XPS) analysis, the biofoulants were removed from the surfaces of the RO membranes with a sterilized soft rubber scrubber (VWR, Denver) and nanopure water. The reproducibility of biofoulant cleaning using this approach was highly reliable, which corresponded to the standard error of estimation during the above analyses. Then, the surfaces were dried at room temperature. Before contact angle and XPS analyses, the RO membrane surfaces were dried again under nitrogen gas for $1 \mathrm{~min}$.

Contact angles, the direct measurement of surface hydrophobicity, were determined by the sessile drop method using a Ramé-Hart contact angle goniometer (Model 100-22, Ramé-Hart Instrument Co., NJ). One to two $\mu 1$ of nanopure water were introduced onto the clean and air dried membrane surface by a Gilmont (IL) microsyringe with a droplet diameter of 0.4 $0.5 \mathrm{~mm}$. The images of water droplets were captured by a CCD camera and analyzed for contact angles (advanced and receding angles) using DROPimage Standard software (Ramé-Hart Instrument Co., NJ). The water droplet diameter on the virgin and air dried cleaned surfaces did not change, so the captive bubble/ drop method was not considered in this study for contact angle measurements. Ten to 15 different measurements of contact angles were carried out for each piece of physically cleaned membrane and its respective virgin membrane surface.

Prior to removal of biofoulants from RO surfaces, XPS analyses of biofoulants on these surfaces were also performed after drying the membranes with biofilm at room temperature. In a previous study (Khan et al. 2010b), there were no changes of surface morphology and chemical compositions resulting from this cleaning procedure of virgin membranes. The XPS analyses of the RO and NF membrane surfaces were done on virgin surfaces and before and after cleaning of biofoulants to characterize the change of surface chemical properties due to the formation of biofoulants and their interactions. The XPS analysis was performed using a 5400 PHI ESCA spectrometer (Physical Electronics, Eden Prairie, MN). The optimized parameters for XPS analysis are described 
elsewhere Khan et al. personal observations. At these operating conditions, the penetration depth of X-ray beam into the sample was approximately $10 \mathrm{~nm}$ and the spot size analyzed was approximately $300 \mu \mathrm{m} \times 700 \mu \mathrm{m}$.

\section{Results}

\section{Reproducibility of biofilm formation (cell distribution and thickness) in replicate experiments, quantitative measurements and visualization of biofoulants on membranes}

Distribution of live and dead cells on the RO and NF surfaces

Figure 1 shows representative images of the orientation of live (green) and dead (red) cells on the top surface of biofoulants formed on RO and NF surfaces. The RO membranes had more organisms, and the relative numbers of live and dead cells were comparable ( $\sim 50 \%$ live and 50\% dead cells). Cells mostly existed as clumps. In contrast, the total number of cells (live and dead) was lower on the top surface of the NF membrane. Also, the proportion of live cells ( 80 to $90 \%$ ) was greater on the NF surface than on the RO membrane. These observations suggest that the NF membrane was less prone to cell accumulation than the RO surface.

In addition to end-point sampling, a time series for biofilm formation was obtained. At the initial sampling time on both surfaces, there were more cells (Figure 1A and D) and the cell numbers decreased by Day- 8 . This suggests that the biofilms on the RO and NF surfaces reached a pseudo-steady state (Figure 1B and E) due to simultaneous attachment and detachment. Lower numbers of cells were heterogeneously distributed across the membrane surfaces on Day-31 (Figure 1C and F) presumably due to shear and detachment. This phenomenon was reproducible and similar trends were
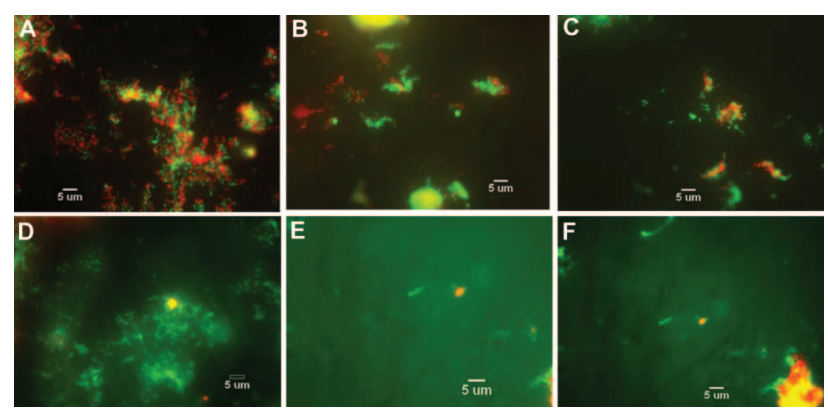

Figure 1. Images and distribution of live and dead cells on the surfaces of RO membrane swatches: (A) and (B) on Day8 and Day-21, respectively, in Phase-II; (C) on Day-31 in Phase-I, and on NF membrane swatches: (D) and (E) on Day-8 and Day-21, respectively, in Phase-II; (F) on Day-31 in Phase-I after staining with Live/Dead BacLight Bacterial Viability Kit. seen in the two replicate experiments even though the runs were different in length (31 vs 28 days).

\section{Quantification of biofoulant thickness and parameters}

Figure 2A and $\mathrm{C}$ shows representative cryo-sectioned images of biofoulants on $\mathrm{RO}$ and NF surfaces. The arrows indicate the position of the membranes underneath the biofoulants. Most of the cells nearest to the membrane surfaces were dead (red) and those near the top regions were mostly alive (green). It appears that the activities of aromatic and semi-aromatic groups on RO and NF surfaces, respectively, were effective up to a certain thickness of biofilm for inactivating the cells in the biofilm. Biofoulants on RO surfaces were more compact than those on NF surfaces. Clusters of cells (live and/or dead) with hollow pockets were observed (Figure 2A and C).

Line scans of these images were used to provide information on the distribution of live and dead cells in the biofouling layer in terms of respective staining intensities and representative images are shown in Figure $2 \mathrm{~B}$ and $\mathrm{D}$. The skewness and intensity of distributions of the regions with live and dead cells depends on the number of cells along the section of biofilm.

The average thickness of live and dead cell regions in the hydrated biofoulants is shown in Figure 3. The accumulated thicknesses of live and dead cells were similar on each day, which is in good agreement with the images obtained with the microscope (Figure 1). However, the average thickness of cells on the NF surface was less than that on RO surface. This
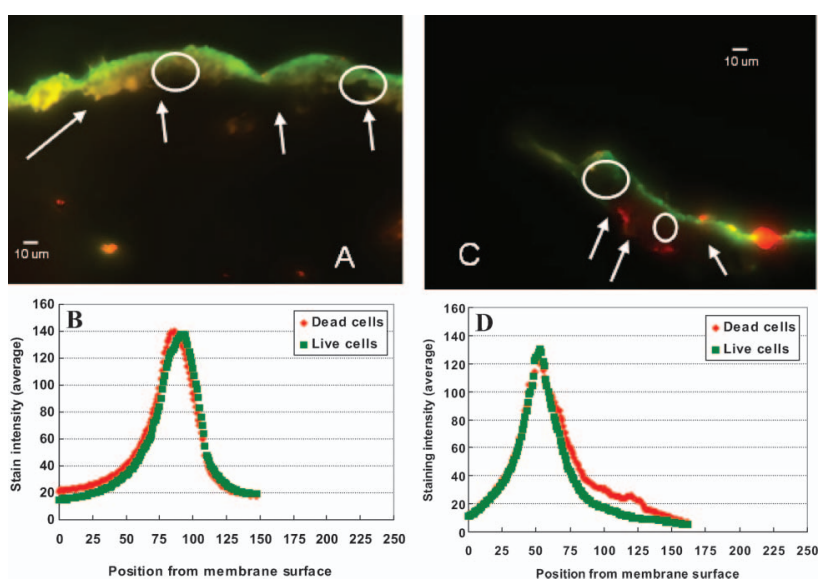

Figure 2. Representative $5 \mu \mathrm{m}$ cryo-sections of (A) RO and (C) NF membranes with biofoulants after staining with the Live/Dead BacLight Bacterial Viability Kit on Day-31 in Phase-I. The profiles of live and dead cell regions in the biofilms on (B) RO and (D) NF membranes after linescanning the respective cryo-sections. The arrows indicate the position of membrane under the biofoulant and circles show the hollow pockets inside the biofoulant. 
difference was significant for Days 8 and $21(p<0.05$ as calculated using a two-tailed Student's $t$-test).

Correlation of biofoulant structure with membrane property changes and quantification of the changes of membrane surface chemical properties due to biofouling formation

Biofilm morphology and its effects on membrane roughness

The morphology of membrane surfaces with and without dehydrated biofoulants was observed with AFM (Figure 4). The morphology of both RO and NF virgin surfaces were heterogeneous (Figure $4 \mathrm{~A}$ and D). After cleaning the biofoulants from the RO and NF surfaces, the morphology of these surfaces were modified.

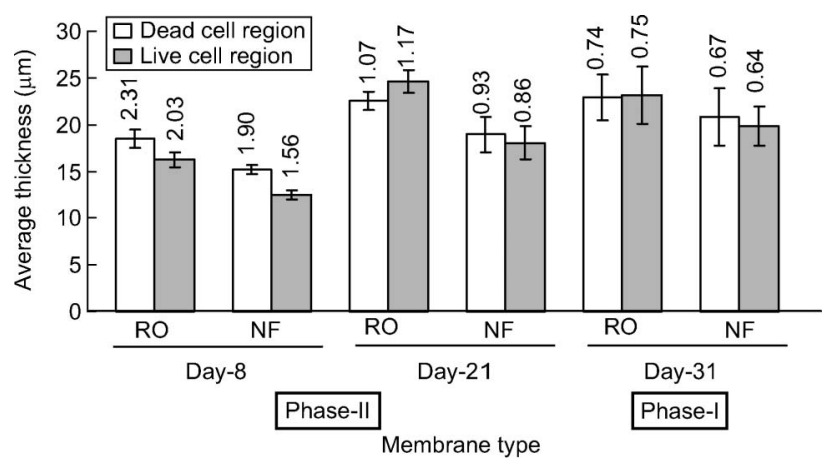

Figure 3. Average thickness of live and dead cells regions in the biofoulants on the surfaces of RO and NF membranes on Day-8 and Day-21 in Phase-II and on Day-31 in Phase-I. Error bars show the \pm standard error (SE) of the mean, which varied from 0.46 to $1.91 \mu \mathrm{m}$. The values on the bar of each live and dead cell region show the average accumulation rate $\left(\mu \mathrm{m} \mathrm{day}{ }^{-1}\right)$ of live and dead cell regions in the biofoulants on the membrane surfaces during operational period in each phase.
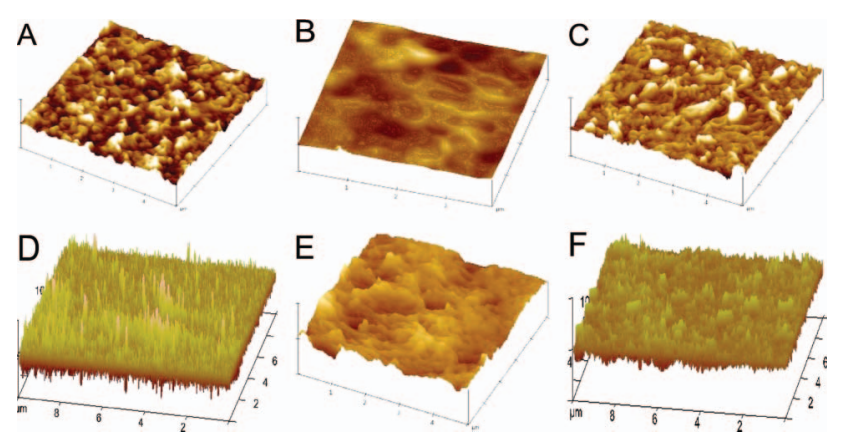

Figure 4. AFM images of: (A) virgin RO membrane, (B) RO membrane with biofoulant, (C) RO membrane after removing biofoulants physically, (D) virgin NF membrane, (E) NF membrane with biofoulant, and (F) NF membrane after removing biofoulants physically on Day-31 in Phase-I.
The average root mean squared (RMS) values of $\mathrm{Rq}$ of virgin surfaces, surfaces with dehydrated biofoulants and surfaces after cleaning the biofoulants were 75.3, 137.5, and $122.6 \mathrm{~nm}$ for RO and 8.2, 59.6 and $47.8 \mathrm{~nm}$ for NF membranes, respectively. The SE of the mean of $\mathrm{Rq}$ for $\mathrm{RO}$ and NF surfaces varied from 4.2 to $5.4 \mathrm{~nm}$ and 3.9 to $4.7 \mathrm{~nm}$, respectively. The accumulated biofoulants on these surfaces were unevenly distributed (Figure 1), which was probably due to the heterogeneous properties of the RO and NF surfaces. Biofouling increased the RO and NF surface roughness up to $48 \mathrm{~nm}$ and $39 \mathrm{~nm}$, respectively.

The AFM images were further characterized by comparing the vertical height profiles along a horizontal line of $10 \mu \mathrm{m}$ for RO and NF surfaces (Figure 5). The 'a-a' sections are selected to describe the maximum peakto-valley distance within the selected area. These sections show the difference in the surface line roughness based on the vertical deviation with respect to the scale of the ordinate and the numbers of peaks and valleys in the specified displacement (ie 'a-a' sections) along the abscissa. Both virgin and cleaned NF surfaces had higher numbers of peaks and valleys in a given distance than those of RO surfaces, while the RO surfaces (virgin and cleaned) had the greatest distance between peaks and valleys compared to those of NF surfaces.

\section{Impact of biofilm on membrane hydrophobicity}

The water contact angles of the virgin membranes and biofouled surfaces after physical cleaning on Day-14 and Day-28 in Phase-II are shown in Figure 6. Taniguchi and Belfort (2002) proposed a correction
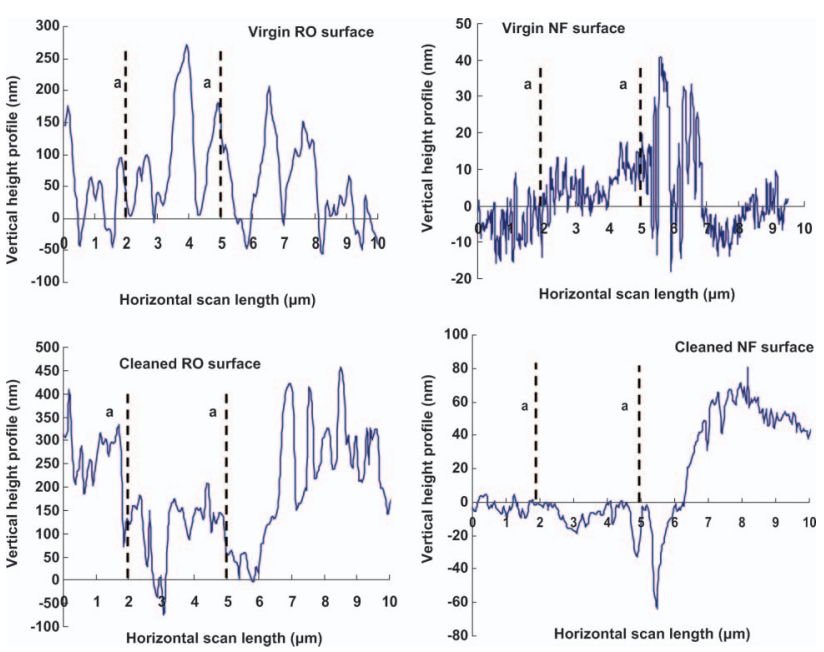

Figure 5. The representative vertical height profiles of virgin and cleaned $\mathrm{RO}$ and $\mathrm{NF}$ membranes taken from line traversing the individual AFM images. The 'a-a' sections are selected to describe the maximum peak-to-valley distance within the selected area. 
factor for the measured contact angle of ultrafiltration (UF) membrane based on the membrane pore size. In this study, the correction factors for contact angles (Figure 6) were not considered, because the pore sizes of these membranes ( $\sim 2 \mathrm{~nm}$ and $\sim 10 \mathrm{~nm}$ for RO and NF membranes, respectively) were much smaller than those of UF membranes $(\sim 100 \mathrm{~nm})$. RO and NF membrane contact angles increased up to $30^{\circ}$ and $4^{\circ}$, respectively, within 28 days due to biofouling formation. The longer colonization time resulted in higher contact angles and greater hydrophobicity. Increases in hydrophobicity are associated with a loss of membrane permeability due to the attached biofouling macromolecules (Herzberg and Elimelech 2007). The contact angle information also suggests that physical cleaning did not adequately remove the biofouling from the membrane surfaces.

Change of membrane chemical elements due to biofilm formation

An increase in contact angle is associated with changes in the surface chemical properties. Representative XPS

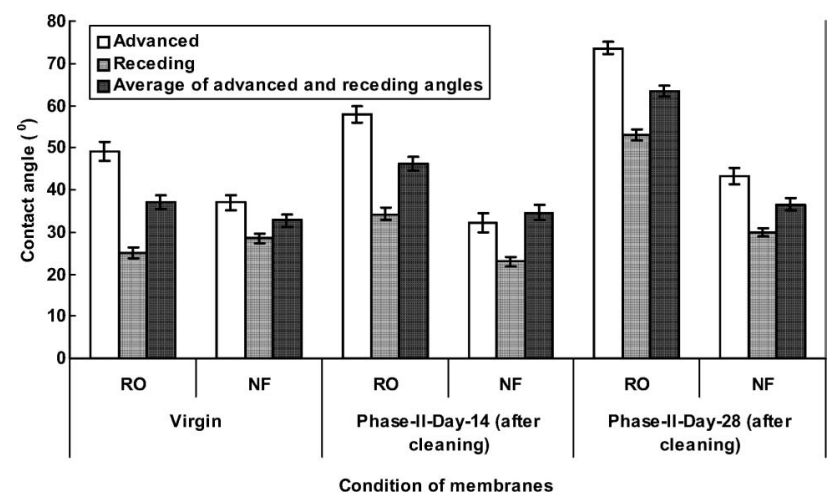

Figure 6. Contact angles of virgin and cleaned $\mathrm{RO}$ and $\mathrm{NF}$ membranes on Day-14 and Day-28 in Phase-II. Error bars show the \pm SE of the mean, which varied from 0.94 to $2.35^{\circ}$. plots of biofoulants on the RO and NF membranes on Day-28 in Phase-II are shown in Figure S1 (see Supporting information [Supplementary material is available via a multimedia link on the online article webpage]). Each element has a characteristic binding energy associated with each core atomic orbital, ie each element will give rise to a characteristic set of peaks in the photoelectron spectrum at kinetic energies determined by the photon energy and the respective binding energies. The presence of peaks at particular energies therefore indicates the presence of a specific element in the sample under study (Ferjani et al. 2000). Table 2 provides the mass percentages of important chemical elements on the RO and NF surfaces before and after cleaning of biofoulants as well as in virgin conditions. Along with biofilm accumulations, inorganic accumulations or the changes in the mass percentage of chemical elements on the RO surfaces were also seen. The increases in calcium, aluminum, and silica on Day28 after cleaning the biofilm could be due to the particulate fouling included in the biofilm or the formation of calcium-aluminum-silicates, which was not confirmed in this study. The incremental change of these three elements was greater for the RO than for the NF surface.

\section{Discussion}

The RO surface accumulated a higher number of cells than the NF surface. This may be related to differences in the chemical composition and characteristics of the membrane surfaces. The active layer of the NF surface was composed of semi-aromatic groups while the RO surface contained aromatic groups. This observation suggests that semi-aromatic groups were more efficient in controlling cell adhesion and biofilm formation (compare Figure 1A and D). Furthermore, there were more dead cells on the RO surfaces; therefore, the aromatic groups were more likely to disinfect the

Table 2. Summary of XPS analyses for the mass percentage of important elements on the surface of virgin RO and NF membranes, biofouled membranes on Day-14 and Day-28, and cleaned and dried surfaces on Day-28 in Phase-II.

\begin{tabular}{|c|c|c|c|c|c|c|c|c|}
\hline & \multicolumn{8}{|c|}{ Important elements $(\%)$} \\
\hline & $\mathrm{C} 1 \mathrm{~s}$ & $\mathrm{O} 1 \mathrm{~s}$ & $\mathrm{~N}$ 1s & P 2s & S $2 p$ & Si $2 \mathrm{~s}$ & $\mathrm{Al} 2 \mathrm{~s}$ & $\mathrm{Ca} 2 \mathrm{p}$ \\
\hline \multicolumn{9}{|l|}{ RO } \\
\hline Virgin surface & 78.9 & 9.2 & 9.4 & 0.3 & 0 & 0.7 & 0 & 0.1 \\
\hline Day-14 (with biofoulant) & 50 & 23.3 & 4.7 & 1.1 & 1.6 & 8 & 1.5 & 0.3 \\
\hline Day-28 (with biofoulant) & 50.6 & 19.5 & 4.1 & 0.6 & 1.1 & 7.1 & 0.8 & 0.6 \\
\hline Day-28 (after cleaning) & 49.6 & 21.8 & 5.1 & 0.8 & 1.4 & 8 & 2.2 & 0.6 \\
\hline \multicolumn{9}{|l|}{ NF } \\
\hline Virgin surface & 67.5 & 13.8 & 13.6 & 0.2 & 0 & 0 & 0 & 0.1 \\
\hline Day-14 (with biofoulant) & 43.6 & 20.9 & 4.6 & 1.1 & 1.6 & 2.9 & 0.7 & 2.1 \\
\hline Day-28 (with biofoulant) & 54.8 & 19.2 & 4.4 & 0.3 & 0.1 & 7.9 & 1.1 & 0.4 \\
\hline Day-28 (after cleaning) & 62.7 & 13.4 & 12.1 & 0 & 0.2 & 1.6 & 0.1 & 0.1 \\
\hline
\end{tabular}


accumulated organisms. As a point of comparison, Vrouwenvelder and van der Kooij (2001) used acridine orange and epifluorescence microscopy to locate cells on $\mathrm{RO}$ and NF biofoulants and found high concentrations of active biomass on the feed side of the membranes in 12 of the 13 treatment plants investigated. In their studies they attributed the biomass accumulation to the biochemical properties of the feed solutions and not specifically related to the membrane properties.

Recent controlled studies show that biofilm formation is a highly regulated process with different bacterial genes being expressed at different stages (Southey-Pillig et al. 2005; Waite et al. 2006). Depending on the nutrient concentrations above the membrane surfaces, the chemotatic responses of planktonic cells are enhanced, which can increase biofilm formation (Herzberg and Elimelech 2008). However, the genes responsible for chemotaxis, motility and other physico-chemical responses of bacteria depend on several parameters of the external environment, which vary dynamically (Waite et al. 2006; Hohne et al. 2009). These phenomena may have contributed to the biofilm development and accumulations, and seen in Figure 1.

Previous work has also shown that under similar aquatic and biological environments, the biofoulant density depends on the membrane surface properties (Khan et al. personal observations). In this work, where RO and NF membranes with different surface properties (aromatic and semi-aromatic surfaces, respectively) were used, it appears that membrane surface chemistry controls biofoulant formation on these surfaces. It is interesting to note that the heterogeneous distribution of biofoulants on these two different membrane surfaces were not identical, although the cell densities based on the top view images at different sampling times followed similar trends. The cell densities were the highest initially and then decreased to a lower level after a pseudo-steady state was attained on both types of membrane.

Furthermore, the peaks of staining intensity (Figure 2B and D) for live cells on the RO and NF surfaces were closer to the biofilm-fluid interface than that for dead cells. In other words, dead cells were in closer proximity to the membrane surfaces and the live cells were at the top of the biofilm. The peak staining intensities of the dead and live cell regions were separated by 2.4 to $5.5 \mu \mathrm{m}$ for the RO surface and 2.3 to $3.2 \mu \mathrm{m}$ for the NF surface. This suggests that the ability of semi-aromatic group on NF surface to disinfect cells was more effective than the aromatic groups on the RO surface and corroborates the microscopy.

Time series analysis also illustrated that the biofouling layers on RO and NF surfaces increased from 8 to $12 \mu \mathrm{m}$ in 2 weeks (from initial sampling on Day-8). The thickness did not continue to increase with longer periods of time (from Day-21 to Day-31), again suggesting that it reached a pseudo-steady state. Also, at Day-31, there was no significant difference as calculated using the $t$-test between the thicknesses of the biofilm on the two different membrane surfaces $(p<0.05)$. Furthermore, there were hollow pockets within the biofilm that are indicative of the spatial variation in porosity within the biofilm (Lewandowski and Beyenal 2005) and such varied porosity distributions will provide different diffusivities that affect membrane performance (Khan et al. 2010b).

The observation on the changes in biofilm thickness with time could be related to both biological and physical parameters in the reactors. In recent studies, Kirisits and Parsek (2007) and Yeon et al. (2009) demonstrated that several disrupting quorum-sensing signals/molecules were responsible at different stages of biofilm formation. However, these studies are strain- and media-dependent and may not be appropriate for the mixed population biofilms used in this study.

It is important to note that based on the microscopic observations, higher cell densities were observed on Day-8 (Figure 1A and D) than those at the end of experiment, Day-21 and Day-31 (Figure 1B, C, E and F). Observations of organisms at the top surface of biofilm obtained using microscopy do not allow for adequate description of the biofilm morphology on membrane surfaces. In contrast, the cryo-sections of biofilm gave much more information about the morphology of the biofoulants and the distributions of live and dead cells on the RO and NF surfaces. It must be noted that these observations are relative and only appropriate for the experimental conditions tested; other experimental conditions may give different results. However, the methods described herein show that relative comparisons between the membranes can be made, and better performance (in terms of biofouling thickness and control) was seen with the NF membrane.

Moreover, membrane surface roughness played an important role in biofoulant accumulation. Biofouling distribution varies across the surface and probably depends on the surface morphology and properties of the membrane (Pang et al. 2005). The rougher surface (RO) was associated with thicker biofouling (Figure 3). Furthermore, cleaning efficiencies were less on the rougher RO surface. The cells and their byproducts, which cause irreversible fouling, could not be completely removed by physical cleaning (Figure 4C and F). Using similar types of RO and NF surfaces with filtration, Kwak and Ihm (1999) reported that RO membranes collected more microbial cells per unit time 
than NF membranes not only because RO membranes were rougher, but also because permeate drag and concentration polarization were more severe. In this study without filtration, similar performances of RO and NF surfaces were observed. Furthermore, rougher membranes have a higher tendency for fouling by natural organic matter (NOM) and soluble microbial products (SMP) (Park et al. 2005).

The vertical height profiles of virgin and cleaned $\mathrm{RO}$ and NF membranes (Figure 5) correspond to the accumulated biofoulants on RO and NF surfaces. Membrane surfaces with higher distances between peaks and valleys after cleaning compared to virgin conditions (RO membranes) indicate that retained biofilm on RO surfaces was more than that on NF surfaces after cleaning. Moreover, the number of peaks and valleys in the 'a-a' section after cleaning on NF surfaces was higher than on the RO surfaces, which indicates that the surface heterogeneities (morphology and roughness) of the NF surfaces were better restored than those of the RO surfaces after cleaning. It has been shown that the surface morphology is important in controlling the accumulation of biofouling (Elimelech et al. 1997; Flemming 1997; Flemming et al. 1997) and at the same time, biofouling can change the surface morphology and roughness (Kwak and Ihm 1999).

In addition, the cause of the changes in hydrophobicity due to biofouling is not well understood. Hypothetically, the bacterial cells in the biofouling layer act as a particulate fouling layer hindering back-diffusion of salts from the membrane surface, thus elevating the osmotic pressure near the membrane surface. Moreover, the EPS component of the biofouling layer seems to mainly increase the biofilm hydraulic resistance to permeate flow (Flemming 2002; Chong et al. 2008). In practice, the coupling effect of increases in osmotic pressure and hydraulic resistance cause a decrease in permeate water flux loss and this effect does not follow a simple gradient along the biofilm. It is not possible to differentiate the individual effects of bacteria and EPS within the biofilm on flux loss. Cells and EPS are randomly distributed in biofoulants on membrane surfaces forming a complex matrix (Flemming et al. 1997). In this study, RO surfaces with higher initial hydrophobicity accumulated more cells (Figures 1 and 6) and had a greater increase in hydrophobicity due to biofouling. This phenomenon was also observed by Park et al. (2005). Even though the average thicknesses of live and dead cell regions on RO and NF surfaces did not increase significantly on Day-31 compared to those on Day-21 (Figure 3), RO surface hydrophobicity due to biofouling continued to increase with time (Figure 6). It is interesting to note that the difference between virgin RO and NF hydrophobicities was $\sim 5^{\circ}$; however, that increased up to $25^{\circ}$ at the end of experiment on Day-28. This observation suggests that the NF surface with semi-aromatic groups was less susceptible to changes in hydrophobicity than the RO surface with aromatic groups.

Field observations have shown that once biofoulants accumulate on membrane surfaces, initial membrane productivity cannot be restored even after chemical (Li and Elimelech 2004) and concentrated salt (Lee and Elimelech 2007) cleaning of biofouled surfaces. The present study without filtration also shows that after biofouling the RO and NF surface hydrophobicities increased; however, the incremental changes would likely be different if the study was performed in filtration mode.

After exposure to the environment in the RDRs, the carbon (C) content on the RO and NF surfaces was reduced by $29 \%$ and $5 \%$, respectively on Day28 (Table 2). The mass-percentage of nitrogen (N) and oxygen $(\mathrm{O})$ decreased and increased, respectively, on the RO membrane in significant percentages, while both decreased on the NF surface in insignificant percentages. The overall changes in these elements were the lowest on the NF surface. The increase in $\mathrm{O}$ and decrease in $\mathrm{C}$ could be due to the presence of EPS, which have high $\mathrm{O}$ to $\mathrm{C}$ ratios (O/C) (Arthur 1989; Riedl et al. 1998). After cleaning the RO surfaces on Day-28, the $\mathrm{O} / \mathrm{C}$ was $\sim 0.44$; however, this ratio on the cleaned NF surface was lower $(\sim 0.21)$. These values suggest that the accumulation of EPS on the RO surface was greater than on the NF surface. As suggested by other observations in this study, these XPS results also suggest that the semi-aromatic NF surfaces were more efficient than aromatic RO surfaces in reducing biofouling accumulation.

The mass percentage ratios of $\mathrm{O}$ and $\mathrm{N}$ for virgin RO and NF surfaces were greater than unity and $\sim 1$. After cleaning, this ratio was $\sim 4$ times and $\sim 1.1$ times higher for the RO and NF surfaces, respectively. These significant changes in the $\mathrm{O}$ and $\mathrm{N}$ mass percentage ratios were likely due to the interaction of different macromolecules in the biofoulants with the membrane (Zhou et al. 2005). It is important to note that the penetration depth of the X-ray beam into the sample was approximately $10 \mathrm{~nm}$ and the chemical elements of cleaned membrane surfaces (in Table 2) may also include the aromatic and semi-aromatic layers of the RO and NF membranes. It is evident from this analysis that biofouling changed the surface chemical compositions in the top layer of the RO and NF surfaces. It is therefore important that RO and NF surfaces should be sufficiently cleaned so that their 
virgin membrane chemistries can be retained after repeated cycles of operation.

\section{Conclusions}

RO and NF membrane filtration enables the production of safe potable water by removing microorganisms and also inorganic and organic compounds, but these membranes always suffer from biofouling. $\mathrm{RO}$ and NF membranes exposed to non-sterile water accumulate biofouling that changes the surface roughness, hydrophobicity, and surface elemental compositions. These changes were observed in a simple, laboratory system without filtration.

The microscopic observations of live and dead cells and biofilm thickness in replicate experiments illustrate that the biofilms on RO and NF surfaces reached comparable pseudo-steady state thicknesses at the end of the run in both phases. Likewise, the distributions of cells on the surfaces of the membranes were similar for both experiments. This illustrates the potential usefulness of a simple model system for producing reproducible biofouling on membrane surfaces.

The changes in membrane properties due to biofouling were less pronounced on the semi-aromatic NF surface than on the aromatic RO surface. The accumulation of biofoulants on the RO surface was greater than on the NF surface. Additionally, the NF surfaces were less prone to bacterial cell and EPS attachment compared to the RO surfaces and the relative number of live cells was greater on the NF surfaces than on the RO surfaces. After cleaning and removal of biofouling, the chemical elements on the NF surfaces and their hydrophobicity were more closely restored to their original states than those on the RO surfaces, which indicates that the NF surfaces were less affected by biofilm formation. Furthermore, biofouling increased the roughness of both membranes, but the NF surface roughness increased less than that of the RO surface.

Towards these goals, the analyses described here provide a comprehensive combination of novel approaches and a suite of analyses that give information that can be compared so that the choices for further membrane testing and evaluation can be based on several measures of membrane performance. The developed approaches can be applied for standard investigations of biofouling and its effect on $\mathrm{RO}$ and NF surfaces used for filtration studies and also for the selection of more effective biofouling resistant surfaces.

\section{Acknowledgements}

This research was supported by the FilmTec Corporation, a subsidiary of the Dow Chemical Company. The authors would like to thank Dr David G. Whitten, Editor-in-Chief of Langmuir and Professor at the Department of Chemical and Nuclear Engineering of the University of New Mexico, for his valuable suggestions and comments on this report.

\section{References}

Arthur SD. 1989. Structure-property relationship in a thin film composite reverse osmosis membrane. J Membrane Sci 46:243-260.

Belfer SJ, Gilron ND, Oren Y. 2005. Comparative study of biofouling of NF modified membrane at SHAFDAN. Desalination 184:13-21.

Burashid K, Hussain AR. 2004. Seawater RO plants operation and maintenance experience: addur desalination plant operation assessment. Desalination 165:11-22.

Chen KL, Song L, Ong SL, Ng WJ. 2004. The development of membrane fouling in full-scale RO processes. J Membrane Sci 232:63-72.

Chong TH, Wong FS, Fane AG. 2008. The effect of imposed flux on biofouling in reverse osmosis: role of concentration polarization and biofilm enhanced osmotic pressure phenomena. J Membrane Sci 325:840-850.

Costerton WJ, Lewandowski Z, Caldwell DE, Korber DR, Lappin-Scott HM. 1995. Microbial biofilms. Annu Rev Microbiol 49:711-745.

Donlan RM. 2002. Biofilms: microbial life on surfaces. Emerg Infect Dis 8:881-890.

Elimelech M, Zhu X, Childress AE, Hong S. 1997. Role of membrane surface morphology in colloidal fouling of cellulose acetate and composite aromatic polyamide reverse osmosis membranes. J Membrane Sci 127:101109.

Ferjani E, Mejdoub M, Roudesli MS, Chehimi MM, Picard D, Delamar M. 2000. XPS characterization of poly (methylhydrosiloxane)- modified cellulose diacetate membranes. J Membrane Sci 165:125-133.

Flemming H-C. 1997. Reverse osmosis membrane biofouling. Exp Therm Fluid Sci 14:382-391.

Flemming H-C. 2002. Biofouling in water systems - cases, causes and countermeasures. Appl Microbiol Biotechnol 59:629-640.

Flemming H-C, Schaule G, Griebe T, Schmitt J, Tamachkiarowa A. 1997. Biofouling- the Achilles heel of membrane processes. Desalination 113:215-225.

Herzberg M, Elimelech M. 2007. Biofouling of reverse osmosis membranes: role of biofilm-enhanced osmotic pressure. J Membrane Sci 295:11-20.

Herzberg M, Elimelech M. 2008. Physiology and genetic traits of reverse osmosis membrane biofilms: a case study with Pseudomonas aeruginosa. ISME J 2:180-194.

Herzberg M, Kang S, Elimelech M. 2009. Role of extracellular polymeric substances (EPS) in biofouling of reverse osmosis membranes. Environ Sci Technol 43:4393-4398.

Hohne DN, Younger JG, Solomon MJ. 2009. Flexible microfluidic device for mechanical property characterization of soft viscoelastic solids such as bacterial biofilms. Langmuir 25:7743-7751.

Ivnitsky H, Katz I, Minz D, Shimoni E, Chen Y, Tarchitzky J, Semiat R, Dosoretz CG. 2005. Characterization of membrane biofouling in nanofiltration processes of wastewater treatment. Desalination 185:255-268.

Kaufman Y, Berman A, Freger V. 2010. Supported lipid bilayer membranes for water purification by reverse osmosis. Langmuir 26:7388-7395. 
Khan MMT, Pyle BH, Camper AK. 2010a. Specific and rapid enumeration of viable but nonculturable and viable-culturable Gram-negative bacteria by using flow cytometry. Appl Environ Microbiol 76:5088-5096.

Khan MMT, Stewart PS, Moll DJ, Mickols WE, Burr MD, Nelson SE, Camper AK. 2010b. Assessing biofouling on polyamide reverse osmosis (RO) membrane surfaces in a laboratory system. J Membrane Sci 349:429-437.

Kirisits MJ, Parsek MR. 2007. Does Pseudomonas aeruginosa use intercellular signalling to build biofilm communities? Cell Microbiol 8:1841-1849.

Kwak SY, Ihm DW. 1999. Use of atomic force microscopy and solid-state NMR spectroscopy to characterize structure property-performance correlation in high-flux reverse osmosis (RO) membranes. J Membrane Sci 158:143-153.

Lee S, Elimelech M. 2007. Salt cleaning of organic-fouled reverse osmosis membranes. Water Res 41:1134-1142.

Lewandowski Z, Beyenal H. 2005. Biofilms: their structure, activity, and effect on membrane filtration. Water Sci Technol 51:181-192.

Li Q, Elimelech M. 2004. Organic fouling and chemical cleaning of nanofiltration membranes: measurements and mechanisms. Environ Sci Technol 38:4683-4693.

Manttari M, Puro L, Nuortila-Jokinen J, Nystrom M. 2000. Fouling effects of polysaccharides and humic acid in nanofiltration. J Membrane Sci 165:1-17.

Pang CM, Liu W-T. 2007. Community structure analysis of reverse osmosis membrane biofilms and the significance of Rhizobiales bacteria in biofouling. Environ Sci Technol 41:4728-4734.

Pang CM, Hong P, Guo H, Liu W-T. 2005. Biofilm formation characteristics of bacterial isolates retrieved from a reverse osmosis membrane. Environ Sci Technol 39:7541-7550.

Park N, Kwon B, Kim IS, Cho J. 2005. Biofouling potential of various NF membranes with respect to bacteria and their soluble microbial products (SMP): characterizations, flux decline, and transport parameters. J Membrane Sci 258:43-54.

Ridgway HF, Flemming H-C. 1996. Membrane biofouling. In: Mallevialle PEOJ, Wiesner MR, editors. Water treatment membrane processes. New York: McGrawHill. p. 6.1-6.62.

Riedl K, Girard B, Lencki RW. 1998. Influence of membrane structure on fouling layer morphology during apple juice clarification. J Membrane Sci 139:155-166.
Schneider RP, Ferreira LM, Binder P, Bejarano EM, Goes KP, Slongo E, Machado CR, Rosa GMZ. 2005. Dynamics of organic carbon and of bacterial populations in a conventional pretreatment train of a reverse osmosis unit experiencing severe biofouling. J Membrane Sci 266:18-29.

Southey-Pillig CJ, Davies DG, Sauer K. 2005. Characterization of temporal protein production in Pseudomonas aeruginosa biofilms. J Bacteriol 187:8114-8126.

Stoodley H-L, Stoodley P. 2002. Developmental regulation of microbial biofilms. Curr Opin Biotech 13:228-233.

Subramani A, Hoek EMV. 2008. Direct observation of initial microbial deposition onto reverse osmosis and nanofiltratio membranes. J Membrane Sci 319:111-125.

Taniguchi M, Belfort G. 2002. Correcting for surface roughness: advancing and receding contact angles. Langmuir 18:6465-6467.

Vrijenhoek EM, Hong S, Elimelech M. 2001. Influence of membrane surface properties on initial rate of colloidal fouling of reverse osmosis and nanofiltration membranes. J Membrane Sci 188:115-128.

Vrouwenvelder JS, van der Kooij D. 2001. Diagnosis, prediction and prevention of biofouling of NF and RO membranes. Desalination 139:65-71.

Waite RD, Paccanaro A, Papakonstantinopoulou A, Hurst JM, Saqi M, Littler E, Curtis MA. 2006. Clustering of Pseudomonas aeruginosa transcriptomes from planktonic cultures, developing and mature biofilms reveals distinct expression profiles. BMC Genomics 7: Article No. 162, p. $1-14$.

Xu P, Drewes JE, Kim T-U, Bellona C, Amy G. 2006. Effect of membrane fouling on transport of organic contaminants in NF/RO membrane applications. J Membrane Sci 279:165-175.

Yeon K-M, Cheong W-S, Oh H-S, Lee W-N, Hwang B-K, Lee C-H, Beyenal H, Lewandowski Z. 2009. Quorum sensing: a new biofouling control paradigm in a membrane bioreactor for advanced wastewater treatment. Environ Sci Technol 43:380-385.

Zhou Y, Yu S, Liu M, Gao C. 2005. Preparation and characterization of polyamide-urethane thin-film composite membranes. Desalination 180:189-196.

Zhu X, Elimelech M. 1997. Colloidal fouling of reverse osmosis membranes: measurements and fouling mechanisms. Environ Sci Technol 31:3654-3662.

Zularisama AW, Ismaila AF, Salimc R. 2006. Behaviors of natural organic matter in membrane filtration for surface water treatment - a review. Desalination 194:211-231. 